Pacific Journal of 


\title{
NOTE ON THE QUADRATIC CHARACTER OF A QUADRATIC UNIT
}

\author{
Duncan A. Buell, Philip A. Leonard and \\ KENNETH S. WILLIAMS
}

\begin{abstract}
Results are obtained concerning evaluations of the quadratic character of real quadratic units of norm -1 .
\end{abstract}

1. Introduction. Let $m$ be a positive squarefree integer, and let $\varepsilon_{m}$ denote the fundamental integral unit of the real quadratic field $Q(\sqrt{m})$, so that $\varepsilon_{m}=T+U \sqrt{m}$ with positive integers $T$ and $U$. Throughout, it is assumed that $\varepsilon_{m}$ has norm -1 , so that $m \equiv 1,5$ or $2(\bmod 8)$, and all odd primes $q$ dividing $m$ satisfy $q \equiv 1(\bmod 4)$. A number of recent papers ([1]- [3], [7], [9]-[12], [14], [16], [17]) have computed the quadratic character of such $\varepsilon_{m}$ modulo a rational prime $p$, in terms of representations of a power of $p$ by positivedefinite binary quadratic forms of a certain discriminant associated with $m$. In this note we prove a result which, among other things, identifies the correct form-discriminant for evaluations of this type. A number of illustrations will be given in $\S 3$ and $\S 4$, after the proof in $\S 2$ of the following theorem.

TheOREM. Let $f=1,2$ or 4 according as $m \equiv 1,5$ or $2(\bmod 8)$. Let $G$ denote the group of primitive positive-definite binary quadratic forms of discriminant $-4 m f^{2}$. Then $G$ contains a subgroup $H$ such that

and

(i) $G / H$ is cyclic of order 4 ,

(ii) the prime $p$ satisfies $(-1 / p)=(m / p)=\left(\varepsilon_{m} / p\right)=1$ if and only if $p$ is represented by a form from a class in $H$.

Before proving this result, we note that an analysis of the equation $T^{2}+1=m U^{2}$ in the ring of Gaussian integers gives the following result.

Lemma. There exist integers $A, B, C, D$ such that $1+T i=$ $(A+B i)(C+D i)^{2}, m=A^{2}+B^{2}, A \equiv 1(\bmod 4)$, and $B \equiv 0,2$ or $T(\bmod 4)$ according as $m \equiv 1,5$ or $2(\bmod 8)$. (Note: $C-1 \equiv D \equiv 0(\bmod 2))$.

2. Proof of the theorem. The splitting field, over $Q$, of the polynomial $x^{4}-2 T x^{2}-1$ is $M=Q\left(i, \sqrt{m}, \sqrt{\varepsilon_{m}}\right)$, which is dihedral over $Q$, and cyclic of degree 4 over $K=Q(\sqrt{-m})$. The primes $p$ 
satisfying $(-1 / p)=(m / p)=\left(\varepsilon_{m} / p\right)=1$ are precisely those which split completely in $M$. It follows (see [4], especially Satz 8, proof of sufficiency) that, for the positive integer $f$ such that $(f)$ is the conductor of the abelian extension $M / K$, the group $G$ of classes of forms of discriminant $-4 m f^{2}$ has a subgroup $H$ with the properties given in the theorem. It remains to show that $f=1,2$ or 4 according as $m \equiv 1,5$ or $2(\bmod 8)$. Besides $M$ and $K$, we shall require $L=Q\left(\sqrt{\varepsilon_{m}}-\sqrt{\varepsilon_{m}^{\prime}}\right)$ and its subfield $k=Q(i)$. For an abelian extension $E / F$ of number fields, we let $d(E / F)$ denote the relative discriminant, $f(E / F)$ denote the finite part of the conductor, and $N_{E / F}(I)$ the relative norm of the ideal $I$ of $E$. Then from the work of Halter-Koch ([5], Satz 7) $f(M / K)=(f)$ for a positive integer $f$. Moreover ([5], Satz 24, $\left.\left(2^{\prime}\right)\right)$, we have

$$
d(L / Q)=d(K / Q) d(k / Q)\{f(M / K)\}^{2}=16 m f^{2} .
$$

On the other hand (see, for example, [13], p. 148), we have

$$
d(L / Q)=\{d(k / Q)\}^{2} N_{k / Q}(d(L / k))=16 N_{k / Q}(d(L / k)) .
$$

Finally, as $\left(\sqrt{\varepsilon_{m}}-\sqrt{\left.\varepsilon_{m}^{\prime}\right)^{2}}=(1-i)^{2}(A+B i)(C+D i)^{2}\right.$ by the lemma, we have $L=Q(i, \sqrt{A+B i})$. Hence, by direct calculation (or see [8], p. 149), we obtain

$$
d(L / k)=2^{\mathrm{e}}(A+B i),
$$

where $e=0,1$ or 2 according as $m \equiv 1,5$ or $2(\bmod 8)$. Appealing to (2.1), (2.2), (2.3) we obtain the evaluation of $f$ stated above.

3. A numerical example. We illustrate the theorem by calculating the subgroup $H$ for the case $m=226$. As $226 \equiv 2(\bmod 8)$, the theorem tells us that the correct discriminant is -128.113 . The group $G$ is of order 32 , and its structure is $C(8) \cdot C(4)$. The 32 classes can be represented by computing the primitive reduced forms of this discriminant. In order for $p$ to be represented by a class from $H, p$ must satisfy $(-1 / p)=(226 / p)=+1$, so by genus theory only 16 of these forms must be examined. By considering primes represented by these farms, and by appealing to the theorem, we find that $H$ is made up of the principal class, together with the classes of the forms $[5, \pm 4,724],[29, \pm 6,125],[32,0,113]$ and $[25, \pm 6,145]$. Since $H$ contains only two ambiguous classes, $H$ is cyclic of order 8 , and $G / H$ is cyclic of order 4, as indicated by the theorem.

4. Conclusion. It is difficult to give $H$ explicitly in general. In spite of this, we can make the theorem explicit in several cases, by considering primitive representations of powers of $p$ by ambiguous 
classes.

Sometimes this is possible when $G$ has a particular structure. For example, if $m=q_{1} q_{2} \cdots q_{r} \equiv 1(\bmod 8), N\left(\varepsilon_{m}\right)=-1$ and the 2-Sylow subgroup of $G$ is of the type $C\left(2^{t}\right) \cdot C(2)^{r-1}$ with $t \geqq 2 . \quad G^{2}$ is the principal genus in $G$, so that $\left[G: G^{2}\right]=2^{r}$ and $G^{2}$ has a unique subgroup of index 2. This subgroup must be $H \cap G^{2}$, for otherwise $H \cap G^{2}=G^{2}$, implying $G^{2} \cong H$ which contradicts the fact that $G / H$ is cyclic of order 4. Thus $H \cap G^{2}$ has order $l=h / 2^{r+1}$, where $h$ denotes the classnumber of $Q(\sqrt{-m})$, and consists of those classes $C$ in $G^{2}$ such that $C^{l}$ is the principal class. A prime $p$ satisfying $(-1 / p)=\left(q_{1} / p\right)=\cdots=$ $\left(q_{r} / p\right)=+1$ is represented by a class from $G^{2}$. This class lies in $H$ if and only if $p^{l}$ is represented by the principal class. Therefore, by the theorem, $\left(\varepsilon_{m} / p\right)=+1$ if and only if $p^{l}=x^{2}+m y^{2}$. This result is due to Parry [14] when $r=1$. When $r \geqq 2$, a large class of examples is provided by choosing $m=q_{1} q_{2} \cdots q_{r}$, where $r=2$ or $r$ is odd, each $q_{i} \equiv 1(\bmod 8)$, and $\left(q_{i} / q_{j}\right)=-1$ when $i \neq j$, since in this situation $\varepsilon_{m}$ has norm -1 [15] and the 2-Sylow subgroup is of the required type [6].

Another example is provided by choosing $m=q_{1} q_{2} \cdots q_{r}$, where $r$ is odd, each $q_{i} \equiv 5(\bmod 8)$ and $\left(q_{i} / q_{j}\right)=-1$ when $i \neq j$. Again $\varepsilon_{m}$ has norm -1 [15] and the 2-Sylow subgroup of the group of form-classes of discriminant $-4 m$ has the structure $C(2)^{r}$. Thus the 2-Sylow subgroup of $G$ has the structure $C(4) \cdot C(2)^{r-1}$, as going from discriminant $-4 m$ to discriminant $-16 m$ doubles the number of classes but introduces no new genera. The "principal genus case" then follows exactly as in the previous example. The remaining cases in this example are covered by a result of Kaplan and Williams [7]. With $h$ as in the previous paragraph, we set $l^{\prime}=h / 2^{r}$, so that $l^{\prime}$ is odd. If $p$ satisfies $(-1 / p)=(m / p)=+1$ then $p^{l^{\prime}}$ is represented by an ambiguous class, and so $p^{l^{*}}=Q x^{2}+Q^{\prime} y^{2}$, where $Q Q^{\prime}=m, Q \equiv 1(\bmod 8)$ and $Q^{\prime} \equiv 5(\bmod 8)$. Then [7] $\left(\varepsilon_{m} / p\right)=+1$ if and only if $y$ is even.

\section{REFERENCES}

1. P. Barrucand and H. Cohn, Note on primes of type $x^{2}+32 y^{2}$, class number, and residuacity, J. reine angew. Math., 238 (1969), 67-70.

2. J. A. Brandler, Residuacity properties of real quadratic units, J. Number Theory, $\mathbf{5}$ (1973), 271-286.

3. - On a theorem of Barrucand, Boll. Un. Math. Ital., 12 (1975), 50-55.

4. G. Bruckner, Characterisienung der galoisschen Zahlkörper, deren zerlegte Primzahlen durch binäre quadratische Formen gegeben sind, Math. Nachr., 32 (1966), 317-326.

5. F. Halter-Koch, Arithmetische Theorie der Normalkörper von 2-Potenzgrad mit Diedergruppe, J. Number Theory, 3 (1971), 412-443.

6. P. Kaplan, Sur le 2-groupe des classes d'ideaux des corps gradratiques, J. reine angew. Math., 283/284 (1976), 313-363.

7. P. Kaplan and K. S. Williams, Quadratic character of quadratic units and representa- 
tions of primes by binary quadratic forms, in preparation.

8. S. Kuroda, Über die Zerlegung rationaler Primzahlen in gewissen nicht-abelschen galo. isschen Körpern, J. Math. Soc. Japan, 3 (1951), 148-156.

9. E. Lehmer, On the quadratic character of the Fibonacci root, Fibonacci Quart., 5 (1967), 135-138.

10. On the quadratic character of some quadratic surds, J. reine angew. Math., 250 (1971), 42-48.

11. - On some special quartic reciprocity laws, Acta Arith., 21 (1972), 367-377. 12. P. A. Leonard and K. S. Williams, The quadratic and quartic character of certain quadratic units, I, Pacific J. Math., 71 (1977), 101-106; II, Rocky Mountain J. Math., 9 (1979), 683-692.

13. W. Narkiewicz, Elementary and Analytic Theory of Algebraic Numbers, Warsaw, 1974.

14. C. J. Parry, Oo a conjecture of Brandler, J. Number Theory, 8 (1976), 492-495.

15. F. Tano, Sur quelques théorèmes de Dirichlet, J. reine angew. Math., 105 (1889), 160-169.

16. H. C. Williams, The quadratic character of a certain quadratic surd, Utilitas Math., 5 (1974), 49-55.

17. K. S. Williams, On the evaluation of $\left(\varepsilon_{q_{1} q_{2}}\right)$, Rocky Mountain J. Math., 10 (1980), 559-573.

Received January 24, 1978. Research of the third author was supported by National Research Council of Canada Grant No. A-7233.

Louisiana State University

Baton Rouge, LA 70803

Arizona State University

Tempe, AZ 85281

AND

Carleton University

Ottawa Ontario, Canada, (K1S 5B6) 


\section{PACIFIC JOURNAL OF MATHEMATICS}

\section{EDITORS}

DONALD BABBITT (Managing Editor)

University of Galifornia

Los Angeles, California 90024

Hugo RossI

University of Utah

Salt Lake City, UT 84112

C. C. MOORE AND ANDREW OGG

University of California

Berkeley, CA 94720
J. DUGUNDJI

Department of Mathematics University of Southern California Los Angeles, California 90007

R. Finn AND J. Milgram Stanford University Stanford, California 94305

\section{ASSOCIATE EDITORS}

R. ARENS

E. F. BECKENBACH

B. H. NEUManN

F. WOLF

K. YOSHIDA

\section{SUPPORTING INSTITUTIONS}

UNIVERSITY OF ARIZONA

UNIVERSITY OF BRITISH COLUMBIA CALIFORNIA INSTITUTE OF TECHNOLOGY

UNIVERSITY OF CALIFORNIA

MONTANA STATE UNIVERSITY

UNIVERSITY OF NEVADA, RENO

NEW MEXICO STATE UNIVERSITY

OREGON STATE UNIVERSITY
UNIVERSITY OF OREGON

UNIVERSITY OF SOUTHERN CALIFONIA

STANFORD UNIVERSITY

UNIVERSITY OF HAWAII

UNIVERSITY OF TOKYO

UNIVERSITY OF UTAH

WASHINGTON STATE UNIVERSITY

UNIVERSITY OF WASHINGTON 


\section{Pacific Journal of Mathematics}

\section{Vol. 92, No. $1 \quad$ January, 1981}

Michael E. Adams and J. Sichler, Lattices with unique complementation . ....1

Walter Allegretto, Positive solutions and spectral properties of second order

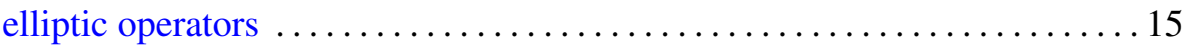

Philip J. Boland and Sean Dineen, Holomorphy on spaces of distribution . . 27

Duncan Alan Buell, Philip A. Leonard and Kenneth S. Williams, Note on

the quadratic character of a quadratic unit $\ldots \ldots \ldots \ldots \ldots \ldots \ldots \ldots \ldots$

Herbert Busemann and Bhalchandra B. Phadke, Two theorems on

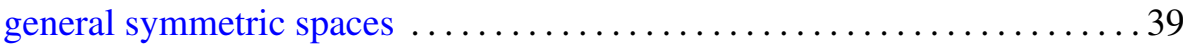

Emeric Deutsch, Bounds for the Perron root of a nonnegative irreducible

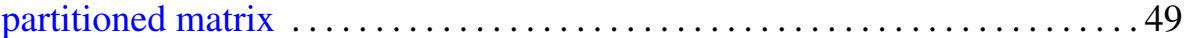

Charles F. Dunkl, A difference equation and Hahn polynomials in two

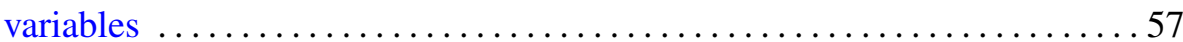

Gustave Adam Efroymson, The Riemann mapping theorem for planar Nash

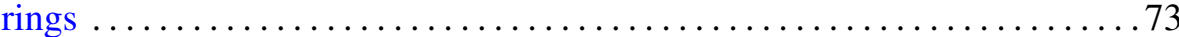

John Albert Fridy and Robert Ellis Powell, Tauberian theorems for matrices generated by analytic functions

Denton Elwood Hewgill, John Hamilton Reeder and Marvin Shinbrot,

Some exact solutions of the nonlinear problem of water waves .......887

Bessie Hershberger Kirkwood and Bernard Robert McDonald, The

symplectic group over a ring with one in its stable range

Esther Portnoy, Transitive groups of isometries on $H^{n}$

Jerry Ridenhour, On the sign of Green's functions for multipoint boundary

value problems

Nina M. Roy, An $M$-ideal characterization of $G$-spaces

Edward Barry Saff and Richard Steven Varga, On incomplete

polynomials. II

Takeyoshi Satō, The equations $\Delta u=P u(P \geqq 0)$ on Riemann surfaces and

isomorphisms between relative Hardy spaces

James Henry Schmerl, Correction to: "Peano models with many generic classes"

Charles Madison Stanton, On the closed ideals in $A(W)$

Viakalathur Shankar Sunder, Unitary equivalence to integral operators

Pavel G. Todorov, New explicit formulas for the $n$th derivative of composite functions

James Li-Ming Wang, Approximation by rational modules on boundary sets

Kenneth S. Williams, The class number of $Q(\sqrt{p})$ modulo 4 , for $p \equiv 5$

$(\bmod 8)$ a prime 